\title{
Expression of breast cancer specific gene-1 (BCSG-1/ $\gamma$-synuclein) is associated with tumour grade but not with clinical outcome of patients with breast cancer
}

\author{
TRACEY A. MARTIN ${ }^{1}$, KELVIN GOMEZ ${ }^{1}$, GARETH WATKINS ${ }^{1}$, \\ ANTHONY DOUGLAS-JONES ${ }^{2}$, ROBERT E. MANSEL ${ }^{1}$ and WEN G. JIANG ${ }^{1}$ \\ ${ }^{1}$ Metastasis and Angiogenesis Research Group, and ${ }^{2}$ Pathology, \\ Wales College of Medicine, Cardiff University, Heath Park, Cardiff, CF14 4XN, UK
}

Received January 26, 2006; Accepted March 13, 2006

\begin{abstract}
This study examined the expression and distribution of BCSG-1 in human breast cancer tissue. IHC revealed that BCSG-1 was primarily seen as a cytosolic protein, weakly staining normal mammary epithelial cells but increased in breast tumour cells. Q-PCR revealed that node negative and positive tumours had similar levels of BCSG-1 transcript and BCSG-1/CK19 ratio. There were significantly higher levels in grade 2 and grade 3 tumours compared to grade 1. Patients with NPI (Nottingham prognostic indicator) $<3.4$, had a predicted $80 \%$ 15-year survival. After a 10-year follow-up, no significant difference was seen between tumours from patients remaining disease-free and those who died of breast cancer. The levels of BCSG-1 significantly correlated with an associated molecule, transglutaminase- $3(\mathrm{r}=0.307, \mathrm{P}<0.05)$, and weakly with transglutaminase-7 $(\mathrm{r}=0.183)$. BCSG-1 is increased in breast tumour cells, is negatively associated with tumour grade and significantly correlates with levels of transglutaminase-3.
\end{abstract}

\section{Introduction}

Synucleins are widely expressed in brain tissue and have been suspected to have links to Alzeimer's disease. Recently, $\gamma$-synuclein (SNCG) has been reported to have a strong connection with cancer.

$\gamma$-synuclein (SNCG) was first found to be similar to a protein known as breast cancer specific gene-1 (BCSG-1), discovered from differential analysis of normal and tumour breast tissues. The molecule was subsequently found to be linked to the proliferation of breast cancer cells, in that over-

Correspondence to: Dr Tracey A. Martin, Metastasis and Angiogenesis Research Group, Wales College of Medicine, Cardiff University, Heath Park, Cardiff, CF4 4XN, UK

E-mail: martinta1@cf.ac.uk

Key words: breast cancer specific gene-1, $\gamma$-synuclein, SNCG, differentiation, breast cancer, metastasis, survival expression of the molecule in breast cancer cells resulted in a faster rate of growth. $\gamma$-synuclein has been shown to be involved with the heat-shock protein (Hsp)-based multiprotein chaperone complex for stimulation of estrogen receptor (ER)- $\alpha$ signalling. It increases the ligand-binding capacity of ER- $\alpha$, and stimulated ligand-dependent activation of ER- $\alpha$ (1). Augmentation of SNCG expression stimulated transcriptional activity of ER- $\alpha$, whereas compromising endogenous SNCG expression suppressed ER- $\alpha$ signalling. The SNCG-mediated stimulation of ER- $\alpha$ transcriptional activity is consistent with its stimulation of ligand-dependent cell growth. Whereas overexpression of SNCG stimulated ligand-dependent cell proliferation, suppression of endogenous SNCG expression significantly inhibited cell growth in response to estrogen $(2,3)$. In clinical breast cancer $(n=79)$, SNCG mRNA, detected using conventional PCR, has been found in $15 \%$ of stage I/II tumours but in $79 \%$ of stage III/IV tumours (4).

In addition to breast cancer, the aberrant expression of BCSG-1 has now been reported in a number of other tumour types, including bladder, pancreas, esophageal, and gastric cancer. However, the conclusions from these studies are controversial. In esophageal tissue, for example, the $\gamma$ synuclein gene was expressed in all 27 cases of normal epithelial tissue, while down-regulation of $\gamma$-synuclein was observed in 16 out of 27 cases $(59.3 \%)$ of esophageal small cell carcinomas. In the same tumour type, an in vitro study concluded that overexpression of $\gamma$-synuclein in cell lines inhibited the growth of the cells (5).

In the current study, we have used quantitative method to quantify the BCSG-1 transcript and correlated the levels of the transcript with clinical parameters and the long-term outcome of patients. We report that, despite the lack of a significant correlation between BCSG-1 and survival and clinical outcome, BCSG-1 is a highly significant differential marker in breast cancer.

\section{Materials and methods}

Materials. RNA extraction and RT kits were obtained from AbGene Ltd. (Surrey, UK). PCR primers were designed using Beacon Designer (CA, USA) and synthesised by Invitrogen Ltd. (Pasley, UK). Molecular biology grade agarose and 
Table I. Clinical details of samples used in this study.

\begin{tabular}{|c|c|}
\hline & $\mathrm{n}$ \\
\hline \multicolumn{2}{|l|}{ Nodal status } \\
\hline Negative & 65 \\
\hline Positive & 55 \\
\hline \multicolumn{2}{|l|}{ ER- $\alpha$ status } \\
\hline Negative & 71 \\
\hline Positive & 49 \\
\hline \multicolumn{2}{|l|}{ ER- $\beta$ status } \\
\hline Negative & 78 \\
\hline Positive & 42 \\
\hline \multicolumn{2}{|l|}{ Grade } \\
\hline 1 & 23 \\
\hline 2 & 41 \\
\hline 3 & 56 \\
\hline \multicolumn{2}{|l|}{ Histology } \\
\hline Ductal & 88 \\
\hline Lobular & 14 \\
\hline \multicolumn{2}{|l|}{ Others } \\
\hline Medullary & 2 \\
\hline Tubular & 2 \\
\hline Mucinous & 4 \\
\hline \multicolumn{2}{|l|}{ TNM staging } \\
\hline TNM 1 & 69 \\
\hline TNM 2 & 40 \\
\hline TNM 3 & 7 \\
\hline TNM 4 & 4 \\
\hline \multicolumn{2}{|l|}{ Clinical outcome } \\
\hline Disease-free & 81 \\
\hline With metastasis & 7 \\
\hline With local recurrence & 5 \\
\hline Died of breast cancer & 20 \\
\hline Died of unrelated diseases & 7 \\
\hline
\end{tabular}

DNA ladder were purchased from Invitrogen. Master mix for routine PCR and customised master mix for quantitative PCR were from AbGene. Rabbit anti-human BCSG-1/ $\gamma$-synuclein polyclonal antibodies were purchased from Santa Cruz Biotechnology Ltd. (Santa Cruz, CA, USA). Peroxidase conjugated anti-rabbit and anti-goat antibodies were from Sigma and a biotin universal staining kit was from Vector Laboratories (Nottingham, UK).

Tissue samples. Breast cancer tissue $(\mathrm{n}=120)$ and normal background tissue $(n=32)$ were collected immediately after surgery and were maintained at $-70^{\circ} \mathrm{C}$ until use. Details of histology and clinical outcome were obtained from pathology reports (Table I). Patients were routinely followed clinically after surgery. The median follow-up period was 120 months. The presence of tumour cells in the collected tissues was verified by a consultant pathologist (A.D.J.), who examined H\&E-stained frozen sections.

Tissue processing, RNA extraction, cDNA synthesis, and $R T-P C R$. Frozen sections of tissues were cut at a thickness of 5-10 $\mu \mathrm{m}$ and were kept for immunohistochemistry and routine histology. A further 15-20 sections were mixed and homogenised using a hand-held homogeniser, in ice-cold RNA extraction solution. The concentration of RNA was determined using a UV spectrophotometer. Reverse transcription was performed using an RT kit with an anchored oligo-dt primer supplied by AbGene, using $1 \mu \mathrm{g}$ total RNA in 96-well plates. The quality of cDNA was verified using B-actin primers. An internal region of BCSG-1 and an internal region which spanned one intron was amplified, the latter as used in the quantitative anlaysis (Table II) together with internal regions for ER, ER- $\beta$ and transglutaminase- 3 and -7 . PCR products were visualised on a $2 \%$ agarose gel and documented using Optimas 6 software.

Quantitative analysis of BCSG-1 transcript. The level of BCSG-1 transcripts, plus ER and ER- $\beta$ transcripts from the above-prepared cDNA was determined using real-time quantitative PCR, based on the Amplifluor ${ }^{\mathrm{TM}}$ technology as we recently reported (6-8), modified from a previously reported method (8). After analysis, the same quantitation was performed for transglutaminase-3 and -7 . Briefly, pairs of PCR primers were designed using the Beacon Designer software (version 2) (sequence given in Table II), but an additional sequence, the $\mathrm{Z}$-sequence (5'-actgaacctgaccg taca-3') which is complementary to the universal $\mathrm{Z}$ probe (Intergen Inc., UK), was added to one of the primers. A Taqman detection kit for $\beta$-actin was purchased from PerkinElmer. The reaction was performed in triplicate using the following: Hot-start Q-master mix (AbGene), 10 pmol of specific forward primer, 1 pmol reverse primer which has the $\mathrm{Z}$-sequence, 10 pmol of FAM-tagged probe (Intergen Inc.), and cDNA from approximate $50 \mathrm{ng}$ RNA. The reaction was performed using IcyclerIQ ${ }^{\mathrm{TM}}$ (Bio-Rad), which is equipped with an optic unit that allows real-time detection of 96 reactions, using the following conditions: $94^{\circ} \mathrm{C}$ for $12 \mathrm{~min}$, 50 cycles of $94^{\circ} \mathrm{C}$ for $15 \mathrm{sec}, 55^{\circ} \mathrm{C}$ for $40 \mathrm{sec}$ and $72^{\circ} \mathrm{C}$ for $20 \mathrm{sec}$. The levels of the transcripts were generated from a standard that was simultaneously amplified with the samples. The levels of transcript are shown in two ways: BCSG-1 transcript and BCSG-1/CK19 ratio, as we have recently reported (9).

Immunohistochemical staining of BCSG-1. This was based on a method we recently described (10). Frozen sections of breast tumours and background tissues were cut at a thickness of $6 \mu \mathrm{m}$ using a cryostat. The sections were mounted on super frost plus microscope slides, air dried and then fixed in a mixture of $50 \%$ acetone and $50 \%$ methanol. The staining procedure was completed simultaneously for all of the sections, to ensure comparison. The sections were then placed in 'Optimax' wash buffer for 5-10 min to rehydrate. Sections were incubated for $20 \mathrm{~min}$ in a $0.6 \%$ BSA blocking solution and probed with the primary antibody (diluted 1:100 for antiBCSG-1). Following washing, sections were incubated for 
Table II. Primer sequences (underlined are Z-sequence for quantitation).

\begin{tabular}{|c|c|c|}
\hline & Sense $\left(5^{\prime}-3^{\prime}\right)$ & Antisense $\left(5^{\prime}-3^{\prime}\right)$ \\
\hline ER & cctactacctggagaacgag & ctcttcggtcttttcgtatg \\
\hline ER- $\beta$ & aaaagaatcattcaatgaca & attaacacctccatccaaca \\
\hline$ß$-actin & atgatatcgccgcgctcg & cgctcgtgtaggatcttca \\
\hline CK-19 quantitation & caggtccgaggttactgac & actgaacctgaccgtacacactttctgccagtgtgtcttc \\
\hline ER quantitation & cctactacctggagaacgag & actgaacctgaccgtacactcttcggtcttttcgtatg \\
\hline ER- $ß$ quantitation & aaaagaatcattcaatgaca & actgaacctgaccgtacaattaacacctccatccaaca \\
\hline TGase-3 quantitation & acaacatcatcttcgtggtt & actgaacctgaccgtacaccttggtagatgaagccata \\
\hline TGase-7 quantitation & agaacgaccacatcacctt & actgaacctgaccgtacatggagagagttggagtcaat \\
\hline B-actin quantitation & atgatatcgccgcgctcg & actgaacctgaccgtacacgctcgtgtaggatcttca \\
\hline
\end{tabular}

$30 \mathrm{~min}$ in the secondary biotinylated antibody (Multilink Swine anti-goat/mouse/rabbit immunoglobulin, Dako Inc.). Avidin Biotin Complex (Vector Laboratories) was then applied to the sections followed by washing to remove excess. Diamino benzidine chromogen (Vector Laboratories) was then added to the sections which were incubated in the dark for $5 \mathrm{~min}$. Sections were counterstained in Gill's haematoxylin and dehydrated in ascending grades of methanol before clearing in xylene and mounting under a cover slip. Staining intensity of BCSG-1 in cytoplasmic area and in nucleus was respectively semi-quantified from non-counterstained images, using a method established in our laboratory (10). Briefly, greyscale digitized images were imported into the Optimas software (Optimas 6.0). Staining intensity was analysed in both nucleus and cytoplasmic compartments. Control staining (without primary antibody) was used for extraction of the background staining. Intensity data were exported to Excel for statistical analysis and are shown here as mean intensity of either the cytoplasmic region or nucleus staining.

Statistical analysis was carried out using the Mann-Whitney U test and the Kruskal-Wallis test, and Kaplan-Meier survival analysis and Cox proportional analysis, where appropriate, using an SPSS (SPSS12) package.

\section{Results}

Distribution of BCSG-1 in human mammary tissue. BCSG-1 was primarily seen as a cytosolic protein that was weakly stained in normal mammary epithelial cells (Fig. 1). No nucleus staining was observed. In breast tumour tissue, there was increased staining in the cytoplasmic region (Fig. 1) but, again, no obvious nucleus staining was seen. In both normal tissue samples, stromal cells displayed no staining.

Levels of the BCSG-1 transcript in normal and tumour tissues. The transcript was determined using quantitative real-time PCR. As shown in Fig. 2, there was no significant difference between normal and tumour tissues. Given the epithelial nature of BCSG-1 staining, as seen in Fig. 1, we have used CK19 transcript to normalise the BCSG-1 transcript. As shown in Fig. 2 (insert), the BCSG-1/CK19 ratio in tumours was significantly higher than in normal mammary tissue.

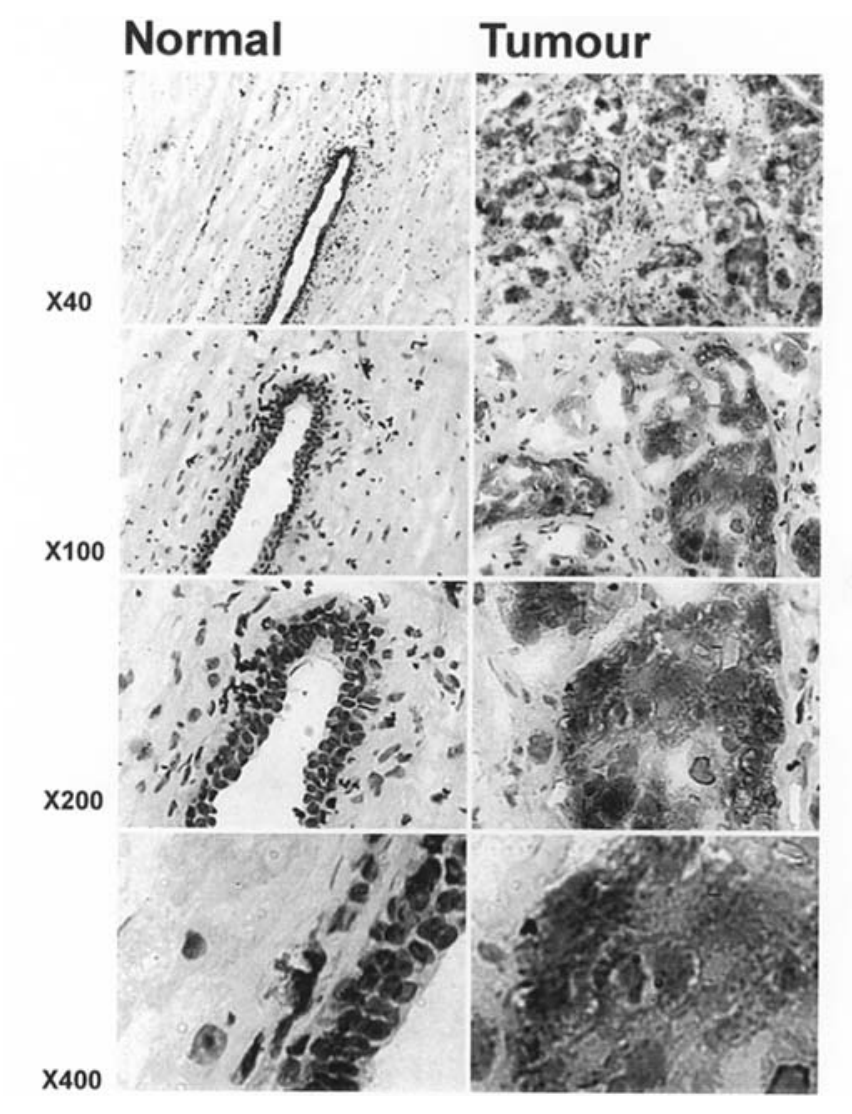

Figure 1. Immunohistochemical staining of BCSG-1 in normal mammary tissue (left) and breast tumour tissue (right). Staining at varying magnifications is shown.

BCSG-1 was associated with tumour differentiation. As shown in Fig. 3, significantly higher levels of BCSG-1 transcript and BCSG-1/CK19 ratio were seen in grade 2 and grade 3 tumours than in grade 1 tumours. In contrast, node negative and node positive tumours had similar levels of BCSG-1 transcript and BCSG-1/CK19 ratio (Fig. 4A). In addition, levels were also assessed according to predicted prognosis, as assessed by the Nottingham prognostic index (NPI). The predicted clinical outcome was assessed using the NPI, which was calculated using the following formula: 


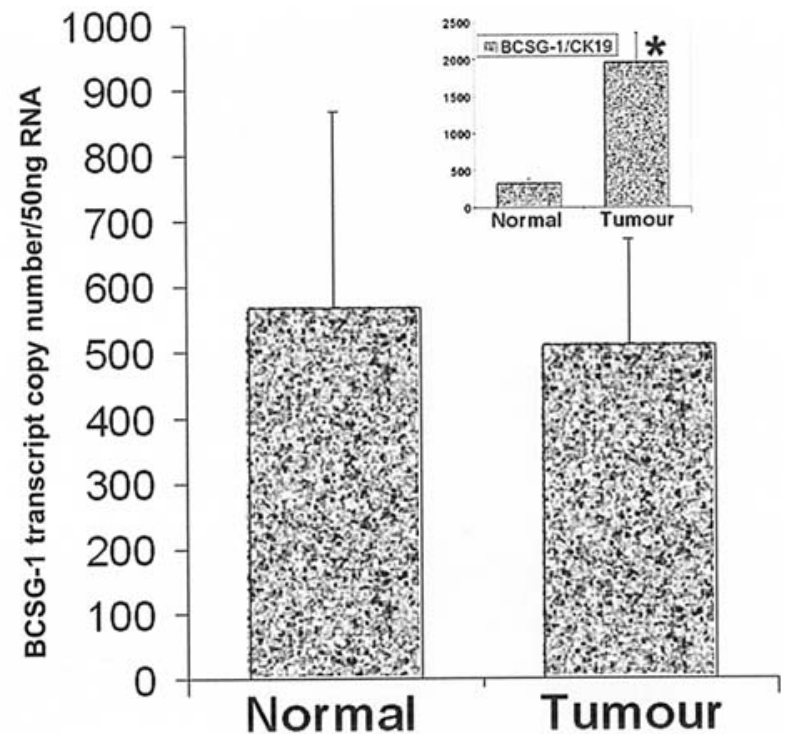

Figure 2. Levels of BCSG-1 transcript in mammary tissues as determined by real-time PCR. No significant difference was seen between normal and tumour tissues. However, after BCSG-1 was normalised by CK19 (insert), tumour tissue had significantly higher levels of BCSG1/CK19 ratio. ${ }^{*} \mathrm{P}=0.0019$.

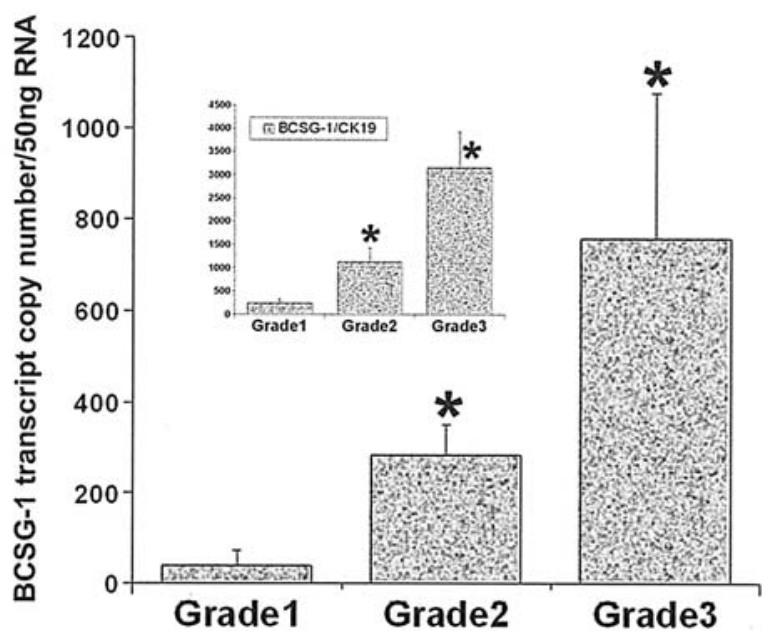

Figure 3. Levels of BCSG-1 transcript and BCSG-1/CK19 ratio (insert) and their relationship with tumour grade. ${ }^{*} \mathrm{P}<0.01$ vs grade 1 .

$\mathrm{NPI}=[0.2 \times$ tumour size $(\mathrm{cm})]+($ tumour grade $)+[$ node status (in order of 1-3)]. Patients with NPI $<3.4$ (coded NPI1 in the current study) had a predicted $80 \% 15$-year survival. Patients with NPI 3.4-5.4 (NPI2) had a predicted $42 \%$ and NPI $>5.4$ (NPI3) had a predicted 13\% 15-year survival, respectively. No significant difference was seen amongst different groups (Fig. 4B), although patients with a poor prognosis had marginally high levels.

No significant link was seen between BCSG-1 and long-term clinical outcome. The current cohort had a 10-year follow-up. As shown in Fig. 5A, there was a marginal rise in BCSG-1 expression in tumours from patients who died of breast cancer. No significant difference was seen between tumours from patients who remained disease-free and from those who
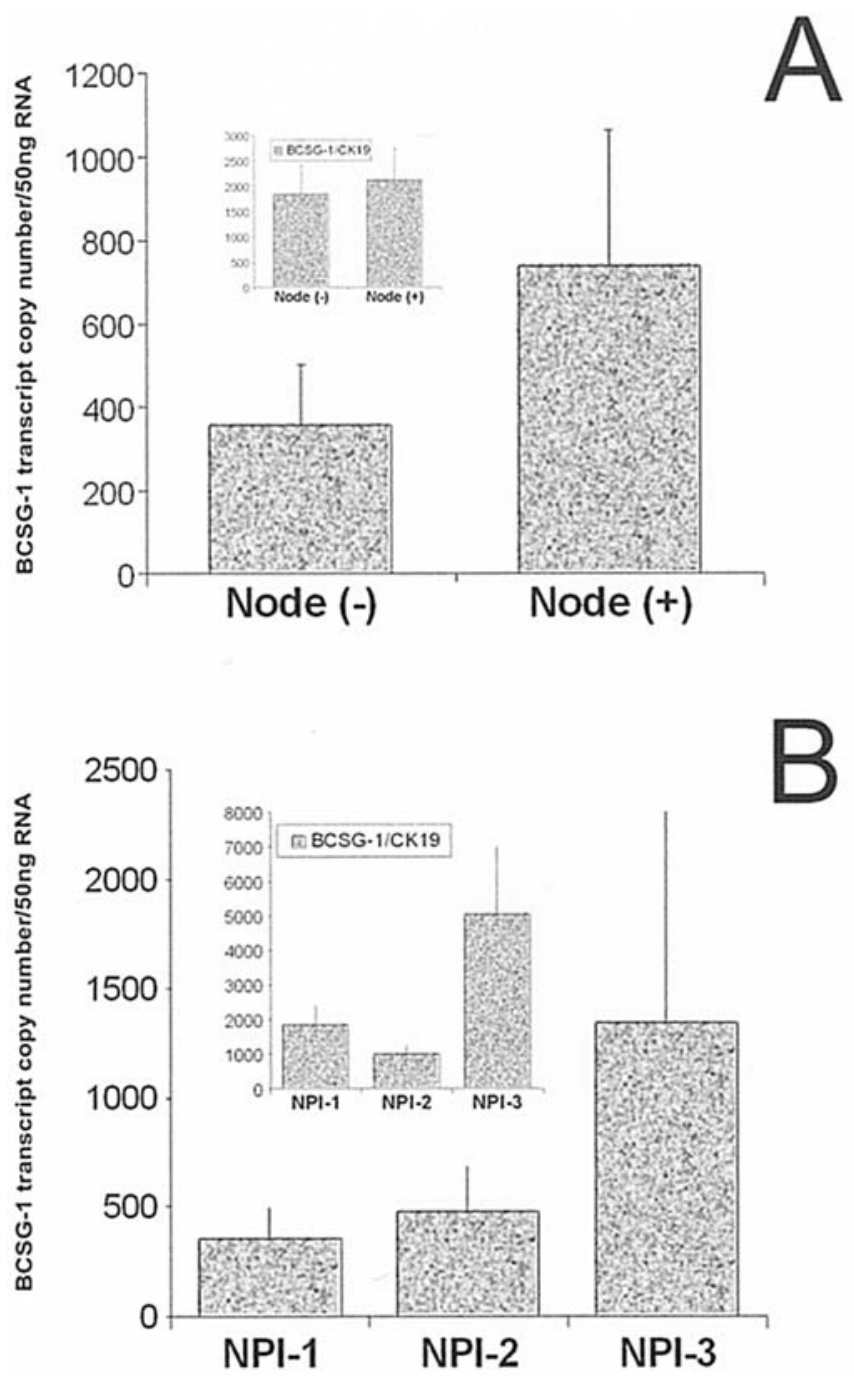

Figure 4. BCSG-1 in node negative and positive tumours (A) and in tumours from patients with different predicted outcomes. (B) BCSG-1 in tumours from patients with different prognoses. Inserts: BCSG-1/CK19 ratio.

died of breast cancer. Kaplan-Meier survival analysis failed to show a significant correlation between BCSG-1 and survival over a 10-year period, although patients with low levels of BCSG-1 had a marginally longer survival time [133.46 (122.9144.0) months, mean and $95 \% \mathrm{CI}]$, compared to those with high levels [127.8 (110.4-145.1) months, $\mathrm{P}=0.06$ ] (Fig. 5B).

We have additionally explored the correlation between BCSG-1 and other molecules that were potentially associated with it. The levels of BCSG-1 significantly correlated with transglutaminase-3 $(r=0.307, \mathrm{P}<0.05)$ and weakly with transglutaminase-7 $(\mathrm{r}=0.183)$. As expected, there was a significant correlation between BCSG-1 and ER- $\beta(r=0.264, \mathrm{P}<0.05$ after CK19 normalisation).

\section{Discussion}

The current study has found that BCSG-1 (breast cancer specific gene-1) or $\gamma$-synuclein (SNCG) is increased in breast cancer tissue, is significantly higher in grade 2 and grade 3 tumours than in grade 1 tumours and significantly correlates with transglutaminase-3, a potentially associated molecule, and ER-B. 
A

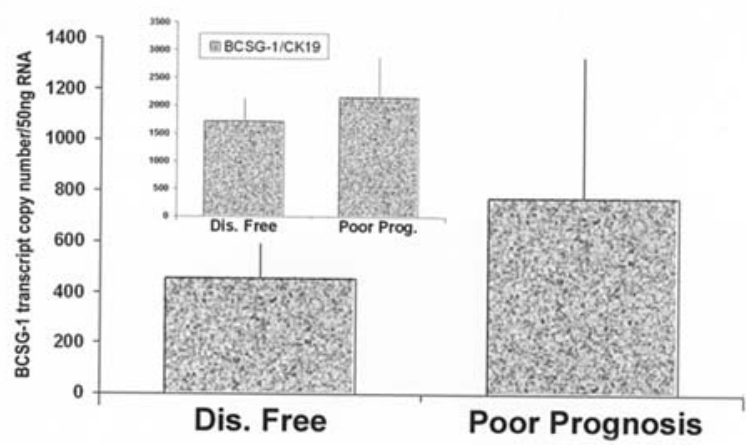

B

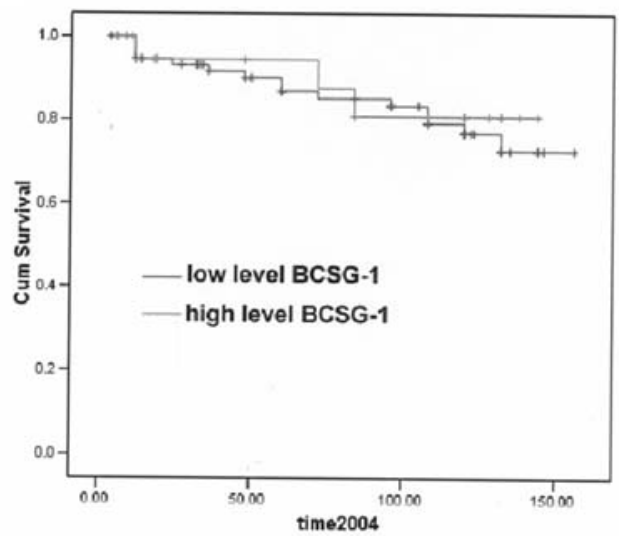

Figure 5. (A) BCSG-1 and the BCSG1/CK19 ratio in tumours from patients with different clinical outcomes. (B) Kaplan-Meier survival curve showing the survival of these patients.

$\gamma$-synuclein (SNCG) was first found to be similar to a protein known as breast cancer specific gene-1 (BCSG-1), discovered from differential analysis of normal and tumour breast tissues. It has been subsequently found not to be breast cancer specific, but found in a range of cancer types. $\gamma$-synuclein has been seen to be a potential marker of human bladder cancer and is raised in urine in a high proportion of patients with bladder cancer (11).

In pancreatic cancer, $\gamma$-synuclein mRNA and protein were found to be overexpressed in a majority of the cell lines (12). The protein was found in over $60 \%$ of pancreatic tumour tissues in stage I and stage II and in $38 \%$ of serum samples. The study has reported that the overexpression of $\gamma$-synuclein correlates with perineural and lymph node invasion (12).

In gastric cancer, $\gamma$-synuclein was also overexpressed, both in cell lines and in primary gastric tumour tissue. This was subsequently found to be linked to the $\mathrm{CpG}$ demethylation of $\gamma$-synuclein (13). It was very interesting to note that demethylation was more frequent in primary gastric cancers positive for lymph node metastasis $(51 \%$; 26 of 51$)$ than in cancers without lymph node involvement $(26 \% ; 14$ of 54 ; $\mathrm{P}<0.05)$, and also more common in stages II-IV (48\%; 27 of 56) than in stage I $(27 \% ; 13$ of 49$)$ cancers $(\mathrm{P}<0.05)$.
This is the first study to use a large cohort of breast tissue samples to determine the range and level of BCSG-1/SNCG in human breast cancer. Although BCSG-1/SNCG levels were increased in tumour tissue, it was interesting to note that this correlated with increasing grade of tumour. Moreover, higher levels of BCSG-1 also correlated with levels of transglutaminase-3. Tissue transglutaminase has been reported to catalyse the cross link of the synucleins and contribute to disease progression in Parkinson's disease $(14,15)$. Western blotting analysis failed to detect any of the synucleins in normal breast and ovarian tissues, but found synucleins in $82 \%$ of latestage breast tumours and $87 \%$ of ovarian tumours (16). This is potentially interesting as it demonstrates a potential link between transglutaminase expression and BCSG-1 expression with breast cancer progression.

Why BCSG-1/SNCG is increased in breast tumour tissue may be due to the methylation status of the gene. Demethylation of exon 1 of BCSG-1/SNCG is an important factor responsible for the aberrant expression of BCSG-1/SNCG in breast carcinoma (17). In human breast and ovarian cancer cell lines, BCSG-1/SNCG exon 1 was hypomethylated which may contribute to the overexpression of BCSG-1/SNCG in these cells (18).

BCSG-1/SNCG may be an indicator of disease progression, although how this protein may be involved remains to be explained. It has been shown that overexpression of $\gamma$ synuclein leads to constitutive activation of ERK1/2 and downregulation of JNK1 in response to a host of environmental stress signals, including UV, arsenate, and heat shock. $\gamma$ synuclein-expressing cells are significantly more resistant to the chemotherapeutic drugs, paclitaxel and vinblastine, as compared with the parental cells. $\gamma$-synuclein may also block apoptosis pathways in cancer cells (19). It is also interesting to note that overexpression of $\gamma$-synuclein resulted in upregulation of MMP-2 and MMP-9 in retinoblastoma cells, possibly by increasing the efficiency of the MMP-9 promoter (20).

In conclusion, BCSG-1 is increased in breast tumour cells and is associated with tumour grade. Moreover, increased levels of BCSG-1 significantly correlate with levels of transglutaminase- 3 and correlate with ER- $B$ expression. We conclude that BCSG-1 increases in human breast cancer; particularly, in association with increased grades of tumour. As it has been shown that overexpression of BCSG-1 leads to resistance to chemotherapy and is linked to disease progression in other diseases, this protein requires further study to elucidate its role in breast cancer.

\section{References}

1. Jiang Y, Liu YE, Goldberg ID and Shi YE: Gamma synuclein, a novel heat-shock protein-associated chaperone, stimulates liganddependent estrogen receptor alpha signaling and mammary tumorigenesis. Cancer Res 64: 4539-4546, 2004.

2. Jia T, Liu YE, Liu J and Shi YE: Stimulation of breast cancer invasion and metastasis by synuclein gamma. Cancer Res 59: 742-771, 1999.

3. Jiang Y, Liu YE, Lu A, Gupta A, Goldberg ID, Liu J and Shi YE: Stimulation of estrogen receptor signaling by gamma synuclein. Cancer Res 63: 3899-3903, 2003.

4. Wu K, Weng Z, Tao Q, Lin G, Wu X, Qian H, Zhang Y, Ding X, Jiang Y and Shi YE: Stage-specific expression of breast cancerspecific gene gamma-synuclein. Cancer Epidemiol Biomarkers Prev 12: 920-925, 2003. 
5. Zhou CQ, Liu S, Xue LY, Wang YH, Zhu HX, Lu N and Xu NZ: Down-regulation of gamma-synuclein in human esophageal squamous cell carcinoma. World J Gastroenterol 9: 1900-1903, 2003.

6. Jiang WG, Watkins G, Lane J, Douglas-Jones A, Cunnick GH, Mokbel M and Mansel RE: Prognostic value of Rho family and and rho-GDIs in breast cancer. Clin Cancer Res 9: 6432-6440, 2003.

7. Jiang WG, Douglas-Jones A and Mansel RE: Level of expression of PPAR-gamma and its co-activator (PPAR-GCA) in human breast cancer. Int J Cancer 106: 752-757, 2003.

8. Jiang WG, Watkins G, Fodstad O, Douglas-Jones A, Mokbel K and Mansel RE: Differential expression of the CCN family members, Cyr61, CTGF and Nov in human breast cancer. Endocr Relat Cancer 11: 781-791, 2004.

9. Jiang WG, Davies G and Fodstad O: Com-1 p8 in oestrogen regulated growth of breast cancer cells, the ER- $\beta$ connection. Biochem Biophys Res Commun 330: 253-262, 2005.

10. Davies G, Jiang WG and Mason MD: Cell-cell adhesion and signalling intermediates in human prostate cancer. J Urol 163: 985-992, 2000.

11. Iwaki H, Kageyama S, Isono T, Wakabayashi Y, Okada Y, Yoshimura K, Terai A, Arai Y, Iwamura H, Kawakita M and Yoshiki T: Diagnostic potential in bladder cancer of a panel of tumor markers (calreticulin, gamma-synuclein, and catecholo-methyltransferase) identified by proteomic analysis. Cancer Sci 95: 955-961, 2004.

12. Li Z, Sclabas GM, Peng B, Hess KR, Abbruzzese JL, Evans DB and Chiao PJ: Overexpression of synuclein-gamma in pancreatic adenocarcinoma. Cancer 101: 58-65, 2004.

13. Yanagawa N, Tamura G, Honda T, Endoh M, Nishizuka S and Motoyama T: Demethylation of the synuclein gamma gene $\mathrm{CpG}$ island in primary gastric cancers and gastric cancer cell lines. Clin Cancer Res 10: 2447-2451, 2004.
14. Andringa G, Lam KY, Chegary M, Wang X, Chase TN and Bennett MC: Tissue transglutaminase catalyzes the formation of alpha-synuclein crosslinks in Parkinson's disease. FASEB J 18: 932-934, 2004.

15. Junn E, Ronchetti RD, Quezado MM, Kim SY and Mouradian MM: Tissue transglutaminase-induced aggregation of alpha-synuclein: implications for Lewy body formation in Parkinson's disease and dementia with Lewy bodies. Proc Natl Acad Sci USA 100: 2047-2052, 2003.

16. Bruening W, Giasson BI, Klein-Szanto AJ, Lee VM, Trojanowski JQ and Godwin AK: Synucleins are expressed in the majority of breast and ovarian carcinomas and in preneoplastic lesions of the ovary. Cancer 88: 2154-2163, 2000.

17. Lu A, Gupta A, Li C, Ahlborn TE, Ma Y, Shi EY and Liu J: Molecular mechanisms for aberrant expression of the human breast cancer specific gene 1 in breast cancer cells: control of transcription by DNA methylation and intronic sequences. Oncogene 20: 5173-5185, 2001.

18. Gupta A, Godwin AK, Vanderveer L, Lu A and Liu J: Hypomethylation of the synuclein gamma gene $\mathrm{CpG}$ island promotes its aberrant expression in breast carcinoma and ovarian carcinoma. Cancer Res 63: 664-673, 2003.

19. Pan ZZ, Bruening W, Giasson BI, Lee VM and Godwin AK: Gamma-synuclein promotes cancer cell survival and inhibits stress-and chemotherapy drug-induced apoptosis by modulating MAPK pathways. J Biol Chem 277: 35050-35060, 2002.

20. Surgucheva IG, Sivak JM, Fini ME, Palazzo RE and Surguchov AP: Effect of gamma-synuclein overexpression on matrix metalloproteinases in retinoblastoma Y79 cells. Arch Biochem Biophys 410: 167-176, 2003. 